PROCEEDINGS OF THE

AMERICAN MATHEMATICAL SOCIETY

Volume 130, Number 10, Pages 3039-3041

S 0002-9939(02)06484-5

Article electronically published on March 14, 2002

\title{
FINITE SUMS OF COMMUTATORS
}

\author{
CIPRIAN POP \\ (Communicated by David R. Larson)
}

\begin{abstract}
We show that elements of unital $C^{*}$-algebras without tracial states are finite sums of commutators. Moreover, the number of commutators involved is bounded, depending only on the given $C^{*}$-algebra.
\end{abstract}

\section{INTRODUCTION}

It was shown in 2] that in finite von Neumann algebras, elements with central trace zero are sums of at most 10 commutators. The $C^{*}$-algebra case was considered in [1]. The main result there states that if the unit of a $C^{*}$-algebra $A$ is properly infinite (i.e. there exist two orthogonal projections $p, q \in A$ equivalent to 1 ), then any hermitian element is a sum of at most five self-adjoint commutators. In this paper we consider the more general case of unital $C^{*}$-algebras $A$ without tracial states and improve the previous result of T. Fack. Note that if the unit of $A$ is properly infinite, then $A$ has no tracial states. The converse is known to be false, at least when $A$ is non-simple (see [4] for further details). $C^{*}$-algebras without tracial states have several nice characterizations, such as [3. This paper also contains another simple proof of the latter result of [3, Lemma 1].

\section{THE RESULT}

Given $a, b \in A$, their commutator is $[a, b]=a b-b a$. A self-adjoint commutator is just a commutator of the form $\left[a^{*}, a\right]=a^{*} a-a a^{*}$.

Theorem 1. Let $A$ be a unital $C^{*}$-algebra. Then the following properties are equivalent:

(1) A has no tracial states.

(2) There exist an integer $n \geq 2$ and elements $b_{1}, b_{2}, \ldots, b_{n} \in A$ such that

$$
\sum_{i=1}^{n} b_{i}^{*} b_{i}=1 \quad \text { and } \quad\left\|\sum_{i=1}^{n} b_{i} b_{i}^{*}\right\|<1 .
$$

(3) There exists an integer $n \geq 2$ such that any element of $A$ can be expressed as a sum of $n$ commutators and any positive element can be expressed as a sum of at most $n$ self-adjoint commutators.

Remark 2. The equivalence of (1) and (2) is just [3, Lemma 1]. As mentioned, in this paper we give a new simple proof.

Received by the editors February 20, 2001 and, in revised form, May 29, 2001.

2000 Mathematics Subject Classification. Primary 46L05.

(C)2002 American Mathematical Society 
Remark 3. The integer $n$ appearing in (2) above automatically satisfies property (3). If the unit of $A$ is properly infinite, there exist two isometries $v_{1}, v_{2} \in A$ with orthogonal ranges. Let $b_{i}=(1 / \sqrt{2}) v_{i}$ for $i=1,2$. Then $b_{1}^{*} b_{1}+b_{2}^{*} b_{2}=1$ and $b_{1} b_{1}^{*}+b_{2} b_{2}^{*} \leq 1 / 2$; thus the property from (2) is achieved with $n=2$. Thus in a properly infinite $C^{*}$-algebra, every element is the sum of two commutators, every positive element is the sum of two self-adjoint commutators, and every self-adjoint element is the sum of four self-adjoint commutators.

Proof. The implication $(3) \Rightarrow(1)$ is trivial.

$(1) \Rightarrow(2)$. Consider

$$
R=\left\{\sum_{i=1}^{s}\left(a_{i}^{*} a_{i}-a_{i} a_{i}^{*}\right) ; s \geq 1, a_{i} \in A\right\}
$$

the set of finite sums of self-adjoint commutators of $A$. Note that $R \subset A_{s a}$ is a real vector subspace of $A_{s a}$. Put $\delta=\operatorname{dist}(1, R)$.

We show that $\delta<1$. Suppose the contrary, i.e. $\delta=1$. This is equivalent to

$$
\|t+x\| \geq|t|, \quad \forall x \in R, \quad \forall t \in \mathbb{R} .
$$

It follows that $\varphi(t+x)=t$ is a real bounded functional on $\mathbb{R} 1+R$ of norm 1 . By the Hahn-Banach theorem it can be extended to a norm-1 functional on $A_{s a}$ and furthermore to a bounded complex functional on $A$, also denoted by $\varphi$. Observe that $\varphi$ is necessarily a tracial state on $A$, which contradicts our hypothesis.

Because $\delta<1$, there exist some elements $a_{1}, a_{2}, \ldots, a_{m} \in A$ such that $t_{0}=$ $\left\|1-\sum_{i=1}^{m}\left(a_{i}^{*} a_{i}-a_{i} a_{i}^{*}\right)\right\|<1$. In particular we have

$$
\sum_{i=1}^{m} a_{i} a_{i}^{*} \leq-1+t_{0}+\sum_{i=1}^{m} a_{i}^{*} a_{i}
$$

Let $k=\left\|\sum_{i=1}^{m} a_{i}^{*} a_{i}\right\|$ and $a_{m+1}=\left(k-\sum_{i=1}^{m} a_{i}^{*} a_{i}\right)^{1 / 2}$. Then we have

$$
\sum_{i=1}^{m+1} a_{i}^{*} a_{i}=k ;
$$

but on the other hand, by (1) we have also

$$
\sum_{i=1}^{m+1} a_{i} a_{i}^{*} \leq-1+t_{0}+k
$$

The required properties are now fulfilled with $n=m+1$ and $b_{i}=(1 / \sqrt{k}) a_{i}$.

$(2) \Rightarrow(3)$. Suppose that $b_{1}, b_{2}, \ldots, b_{n}$ are as in (2). Define $\Phi(a)=\sum b_{i} a b_{i}^{*}$. Then $\Phi$ is a bounded positive map on $A$ with norm $\|\Phi\|=\left\|\sum b_{i} b_{i}^{*}\right\|<1$. It follows that $I d_{A}-\Phi$ is invertible in the Banach algebra $\mathcal{B}(A)$ of bounded maps on $A$. Let

$$
\Psi=\left(I d_{A}-\Phi\right)^{-1} \text {. }
$$

Note that $\Psi=\sum_{i=0}^{\infty} \Phi^{i}$, thus $\Psi$ is positive too. By definition of $\Psi$, for any $a \in A$ we have

$$
\begin{aligned}
a & =\left(I d_{A}-\Phi\right)(\Psi(a))=\Psi(a)-\sum_{i=1}^{n} b_{i} \Psi(a) b_{i}^{*} \\
& =\sum_{i=1}^{n} b_{i}^{*} b_{i} \Psi(a)-\sum_{i=1}^{n} b_{i} \Psi(a) b_{i}^{*}=\sum_{i=1}^{n}\left[b_{i}^{*}, b_{i} \Psi(a)\right],
\end{aligned}
$$


so $a$ is a finite sum of at most $n$ commutators. If moreover $a$ is a positive element in $A$, then

$$
\begin{aligned}
a & =\left(I d_{A}-\Phi\right)(\Psi(a))=\Psi(a)-\sum_{i=1}^{n} b_{i} \Psi(a) b_{i}^{*} \\
& =\sum_{i=1}^{n} \Psi(a)^{1 / 2} b_{i}^{*} b_{i} \Psi(a)^{1 / 2}-\sum_{i=1}^{n} b_{i} \Psi(a) b_{i}^{*}=\sum_{i=1}^{n}\left[\Psi(a)^{1 / 2} b_{i}^{*}, b_{i} \Psi(a)^{1 / 2}\right],
\end{aligned}
$$

so $a$ is a finite sum of at most $n$ self-adjoint commutators.

\section{Questions}

For an infinite $C^{*}$-algebra $A$ (in the sense that it admits no tracial states) let $\nu(A)$ be the least positive integer such that any element of $A$ is a sum of at most $\nu(A)$ commutators. In all the examples that we know of, we have $\nu(A)=2$. We believe that it is unlikely to always be the case.

In [3] it was shown that if $A$ is an unital exact $C^{*}$-algebra, then there exists an integer $m$ such that $\mathbb{M}_{m}(A)$ is properly infinite. It follows that $\nu\left(\mathbb{M}_{m}(A)\right)=2$. Then a simple computation shows that $\nu(A) \leq 2 m^{2}$. It would be interesting to answer the inverse problem, that is: assuming $\nu(A)$ is known, estimate the least positive integer $m$ such that $\nu\left(\mathbb{M}_{m}(A)\right)=2$.

\section{REFERENCES}

1. Thierry Fack, Finite sums of commutators in $C^{*}$-algebras, Ann. Inst. Fourier (Grenoble) 32 (1982), no. 1, vii, 129-137. MR 83g:46051

2. Thierry Fack and Pierre de la Harpe, Sommes de commutateurs dans les algèbres de von Neumann finies continues, Ann. Inst. Fourier (Grenoble) 30 (1980), no. 3, 49-73. MR 81m:46085

3. Uffe Haagerup, Quasitraces on exact $C^{*}$-algebras are traces, Manuscript distributed at the Operator Algebra Conference in Istanbul, July 1991.

4. Mikael Rørdam, On sums of finite projections, Operator algebras and operator theory (Shanghai, 1997) Contemporary Math. 228, Amer. Math. Soc., Providence, RI, 1998, pp. 327-340. MR 2000a:46098

I.M.A.R., CP 1-764, Bucharest, Romania

Current address: Department of Mathematics, Texas A\&M University, College Station, Texas $77843-3368$

E-mail address: cpop@math.tamu.edu 